\title{
Role of Precision Medicine in Cancer Therapy
}

\section{Preethi $\mathbf{G}^{1}$, Kavitha $\mathbf{S}^{2}$, Dinesh Premavathy ${ }^{3}$, V. Vishnupriya ${ }^{4}$, Gayathri $\mathbf{R}^{5}$}

\section{IJCRR}

Section: Healthcare

Sci. Journal Impact

Factor: $6.1(2018)$

ICV: 90.90 (2018)

(c) (i) (8)

Copyright@IJCRR
'Saveetha Dental College, Saveetha Institute of Medical and Technical Sciences, Saveetha University, Chennai 77, India; '2Lecturer, Department of Biochemistry, Saveetha Dental college, Saveetha Institute of Medical and Technical Science, Saveetha University, Chennai 77, India; ${ }^{3 S e n i o r}$ Lecturer, Department of Anatomy, Saveetha Dental College, Saveetha Institute of Medical and Technical Science, Saveetha University, Chennai 77, India; 4Professor, Department of Biochemistry, Saveetha Dental College, Saveetha Institute of Medical and Technical Science, Saveetha University, Chennai 77, India; ${ }^{5}$ Assistant Professor, Department of Biochemistry, Saveetha Dental College, Saveetha Institute of Medical and Technical Science, Saveetha University, Chennai 77, India.

\section{ABSTRACT}

Cancer, which is also called malignancy, is an abnormal growth of cells. There are more than 200 types of cancers including skin cancer, lung cancer, breast cancer, colon cancer, prostate cancer, lymphoma etc....Cancer treatments are varying like chemotherapy, radiation therapy, surgery etc... but these can be effective only in a subset of the population. Thus, the inherent variability of cancer has led itself to the growing field of precision medicine. Precision medicine plays a major role in cancer therapy. Precision medicine involves the usage of genetic changes in a patient's tumor to determine the type of treatment one has to undergo. Precision medicine is an approach to patient care that allows doctors to select treatments that help patients based on a genetic understanding of their disease. This review highlighted the importance and role of precision medicine in cancer therapy.

Key Words: Cancer, Precision medicine, Chemotherapy, Genetic changes, Chemotherapy

\section{INTRODUCTION}

Cancer is a devastating disease when treatment like chemotherapy and radiation are effective only in a subset of the population. ${ }^{1}$ The inherent variability of cancer has lent itself to the growing field of precision and personalised medicine. ${ }^{2}$ Precision medicine involves testing DNA from a patient's tumour to identify the mutation or other Genetic changes that drive their cancer. They are targeted on patients whose tumours or a specific gene mutation. These are blocked by certain drug compounds. ${ }^{3}$ Ten years ago, researchers in Dana Farber Cancer Institute published that in non-small cell lung cancer patients, tumours had a mutated version of a protein. The epidermal growth factor receptor EGFR responded to a drug that specifically targets the EGFR protein. ${ }^{4}$ Patients with HER2 positive breast cancer survivors for 2 to 3 years who received the targeted therapy with the drug trastuzumab which is also known as Herceptin. ${ }^{4}$ Technologies supporting precision medicines CRISPR/ cas Technology (gene editing), cryo-electron microscopy (cryoEM ) in which the samples are examined at extremely low temperature After decades of research, scientists understand that patients' tumors have genetic changes that cause cancer cells to grow and spread to various organs.
As deeper understanding of tumor and rapid advances in technology is taking place, there are enormous opportunities to create a future for precision medicine in oncology which can be highly specific with minimal toxic effect on patients. Development of precision medicine for cancer patients improves their quality of life. It creates a wider ability of the doctors to use the patient's genetic and other molecular information. Precision medicine helps in avoidance of unnecessary treatment, has lesser side effects or trauma, extended survival rate and reduction in total cost of treatment. ${ }^{5}$

\section{CANCER AND ITS TYPES}

Cancer is an abnormal growth of cells which can arise from any organ or body structure which causes tumour, lesion or lump. Risk of cancer is multiplied in those whose immune system is suppressed due to factors like chronic stress, old age, chronic debilitating disease, previous use of chemotherapy, abuse of drugs such as analgesics, Antibiotics and corticosteroids. ${ }^{6}$ There are various types of cancer like colon cancer, breast cancer, prostate cancer, lung cancer, pancreatic cancer, skin cancer and cervical cancer. ${ }^{7}$ In women the highest cancer prevalence is breast cancer, lung cancer,

\section{Corresponding Author:}

Kavitha S, Lecturer, Department of Biochemistry, Saveetha Dental College, Saveetha Institute of Medical and Technical Science, Saveetha University, 160, Poonamallee High Road, Chennai 600077, Tamilnadu, India;

Telephone: 9567263096; Email: kavithas.sdc@saveetha.com

ISSN: 2231-2196 (Print)

Received: 25.09 .2020
ISSN: $0975-5241$ (Online)

Revised: 18.10 .2020
Accepted: 24.11 .2020
Published: 26.12 .2020 
pancreatic cancer, bronchus, colon and rectum cancer, uterine corpus and thyroid. In children, the highest prevalence is blood cancer, cancers related to brain and lymph nodes. ${ }^{8}$ Many pharmaceutical studies have been on various cancers like oral cancer, hepatic cancer, oral epidermal cancers etc... Several types of cancer can form in the liver. The most common type of liver cancer is hepatocellular carcinoma. There are other types of liver cancers like intrahepatic cholangiocarcinoma and hepatoblastoma which are less common. Oral cancer is one of the common cancers. Generally, the defective apoptosis (programmed cell death) results in enhanced growth in most of the cancer.

\section{TREATMENTS OF CANCER}

There are several treatments for cancer like surgical removal, chemotherapy or other types of cancer specific medication , hormonal therapy and radiation therapy some cancer cells shrink and disappear on their own like in kidney cancer, targeted therapy and radiotherapy. Other treatments include nano medicine, extracellular vesicles for cancer diagnosis and therapy, natural antioxidants in cancer therapy, targeted therapy and immunotherapy, gene therapy for cancer treatment, thermal ablation and magnetic hyperthermia, recent innovations like radiomics and pathomics. ${ }^{9-13}$ Natural medicines which are rich sources of antioxidants. Antioxidants have the potential to suppress cancer and reduce the risk of cancer. Vitamin A, C, A has been reported to reduce the risk of lung cancer. ${ }^{14}$ Sometimes proteolytic enzymes are also used in the medical field especially in cancer therapy. ${ }^{15} \mathrm{Re}$ cently nanomedicine has gained a crucial role in cancer treatment. ${ }^{16}$ Some studies have shown that photosynthesized gold nanoparticles induce apoptosis in cervical cancer cells. ${ }^{17} \mathrm{An}$ other study proved that Sesame induces apoptosis in thyroid cancer cells. ${ }^{18}$ Nanoparticles from Marsdenia tenacissima induce apoptosis in laryngeal cancer. ${ }^{19}$ Thus nanomedicine, precision medicine, gene therapy etc. are the upcoming treatments in cancer showing better cure.

\section{Failure of treatment}

Failure of treatment can be due to varying reasons like unavailability of effective biomarkers, sometimes cancer diagnosis and prognosis becomes difficult. Limitations include conventional chemotherapeutic agents. Metastasis and angiogenesis are a huge problem. Targeting cancer stem cells is difficult. Unresolved inflammation facilitates immune evasion. Growth promotion of cells causes induction of neoplasia where precancerous polyp formation occurs. ${ }^{20}$. Radiation and chemotherapy are the standard treatment for cancer which works by attacking stem cells rapidly in our body. They do not differentiate between healthy cells and cancerous cells. Thus, these treatments cause adverse side effects and often lead to failure in treating cancer.

\section{Precision medicine}

Due to the unpredictable nature of cancer and failure possibilities of other treatments, precision medicine can play a major role in cancer. ${ }^{21}$ The two major roles of precision medicine are deep genome and transcriptome sequencing which helps in facilitating the discovery of molecular players which are involved in cancer onset to identify more reliable biomarkers. Advances in the development of genetic tests has created a potential to transform and create the ability to detect, prevent and treat certain diseases. Therapy approaches which are based on genetic variants and specific biomarkers have been increasing over the last few decades in association with the increasing availability and affordability of genomic sequencing technology. There has been growing interest in and advocacy for precision medicine approaches. ${ }^{22}$

\section{Precision medicine versus personalized medi- cine:}

According to the National research Council, 'personalise medicine' is an older term but it means similar to Precision medicine. But the concern is that the word personalised is misinterpreted to imply the treatment and prevention. ${ }^{23}$ Precision medicine is developed uniquely for each individual and focuses on identifying which approach is effective on patients based on genetic and environmental factors. ${ }^{24}$ The best example for personalised medicine is CYP 450 enzyme and its application to Coumadin therapy (medication used as anticoagulant to treat blood clots). A common example for precision medicine is gleevec drug which is used to treat leukaemia only when the cancer cells have a particular type of genetic makeup.

\section{Advantages of precision medicine}

Precision medicine avoids the unnecessary treatment, sideeffects, trauma, has an extended survival rate and wider adoption. ${ }^{25}$ It involves collecting patients genetic and molecular information for routine medical care. It has an improved ability to predict which treatment is best for a specific patient. It has a better understanding and underlying mechanism for various diseases. Precision medicine has an improved approach for preventing, diagnosing, and treating a wide range of diseases. For better integration, electronic health records are being used to access the medical data easily. ${ }^{26}$

\section{Precision medicine in cancer therapy}

Each individual has their own unique genome, which may include small single nucleotide polymorphisms (SNPs) and/or large changes in DNA nucleotide sequence (mutations). These may take over but also can initiate during a person's lifetime through external agents (e.g. carcinogenic chemicals or radiation). It is usually harmless to the wellbeing of the individual, these genetic modifications can affect the way the body responds to a therapeutic agent either 
through differences to the drug target, or through ADMET considerations (i.e. absorption, distribution, metabolism, excretion and toxicology).

Precision medicine, sometimes referred to as 'personalized medicine' and abbreviated to 'PM', could be a term that's increasingly being employed to explain treatments, including therapeutic agents, tailored to individual patients or groups of patients. The goal is to match therapies to individuals to confirm that they receive effective treatment with minimal toxicity. this is often particularly important for cancer patients who may have a limited lifetime. It is a more individualised procedure based on genetic changes in a person's cancer. There is no need of undergoing repeated cycles of chemotherapy and radiation therapy. There are several drugs which have been approved by the Food and drug administration in the US where the drugs target genetic changes like cells, based on type and the region of spread of cancer. ${ }^{27}$

\section{Precision medicine in breast cancer}

Breast cancer is one of the leading cancers in women. The factors of breast cancer can be genetic, environmental, behavioural like diet, exercise and lifestyle. Increasing evidence of personal genomics susceptibility is the major factor of intervention and prevention..$^{25}$ The predominant breast cancer is associated with expression of oestrogen receptor which is used as a predictive marker. ${ }^{22}$ Two main types of breast cancers are the inter tumour heterogeneity where the cancer arises from the site of same origin and the other type is spatial and temporal intra tumour heterogeneity. In this, there is a reflecting increase of variability in gene mutation in a single tumour. ${ }^{29,30}$

\section{Precision medicine in colon cancer}

Genetics and epigenetics in colon cancer is characterised by colonoscopy screening which detects the mutation and microsatellite instability, hyper methylation in tumours in response to treatment. ${ }^{25}$ Patients with stage one CRC receive colonoscopic polypectomy, endoscopic mucosal resection and for more advanced stages, surgical resection is done. ${ }^{30}$ Among the certain types of cancers, solid tumors with interpatient heterogeneity witness a paradigm shift in the management plan using precision medicine which is more likely to happen. Colorectal cancer (CRC) which is very heterogeneous could be a model disease to execute the concept of precision medicine.

\section{Precision medicine in lung cancer}

There are two main types of lung cancer - non-small cell lung cancer and small cell lung cancer non-small cell lung cancer. Small cell lung cancer occurs in $20 \%$ of the patients. A drug called Crizotinib which is an inhibitor for anaplastic lymphoma kinase shows promising results. Another drug called erlotinib also shows significant results. Non-small cell lung cancer depends on pathological findings. Targeted therapy is based on molecular profiling and tissue preservation. ${ }^{31}$

\section{DISCUSSION}

Cancer is an uncontrolled growth of abnormal cells. Glioma is the primary cause of cancer in most of the people. ${ }^{32}$ There are about 200 types of cancer. Cancer symptoms are different in different cancers. They can be fatigue, weight loss, pain, skin changes like photoaging change in bowel, unusual bleeding, persistent cough, fever and lumps. Research shows that excess body fat increases the risk for several cancers like colorectal cancer, pancreatic cancer etc. Consumption of high dietary fat results in increased fat and storage and can lead to metabolic disorders like stroke, diabetes etc. ${ }^{33}$ There are certain cancers which can be specific for a particular gender or age group. Many studies and researchers have been widely done on this. Usually, treatment of cancer or chemotherapy, radiation therapy and surgery. But new technologies are making a difference in fighting against cancer. Precision medicine is the future hope of the growing field as it involves the testing of DNA to identify the mutation in a patient's tumour. Adaptation to precision and personalised medicine in cancer can bring a great change and can increase the survival rate in many cases.

\section{CONCLUSION}

Research has moved beyond sequencing and it has accurately linked the information of individual patients to receive better outcomes. Treatment responses to precision medicine are highly positive and have an increased survival rate. It helps patients based on the genetic understanding of the disease. The future use of precision medicine can be a better and efficient way to treat cancer and other deadly diseases.

\section{Acknowledgment: None}

\section{Conflict of Interest: Nil}

\section{Source of Funding: Nil}

\section{REFERENCES}

1. Wyatt D and Hulbert-Williams N. Cancer and Cancer Care. SAGE 2015.

2. Seyhan AA, Carini C. Are innovation and new technologies in precision medicine paving a new era in patients centric care? J Transl Med 2019:17.

3. Gameiro GR, Sinkunas V, Liguori GR, et al. Precision Medicine: Changing the way we think about healthcare. Clinics 2018;73: e723.

4. König IR, Fuchs O, Hansen G, et al. What is precision medicine? Euro Resp J 2017;50(4):1700391. 
5. Wong AH-H and Deng C-X. Precision Medicine for Personalized Cancer Therapy. Int J Biol Sci 2015; 11(12):1410-1412..

6. Hanahan D, Weinberg RA. Hallmarks of cancer: the next generation. Cell 2011;144(5):646-674.

7. Roy PS, Saikia BJ. Cancer and cure: A critical analysis. Indian J Cancer 2016;53(3):441-442.

8. Hassanpour SH, Dehghani M. Review of cancer from perspective of molecular. J Cancer Res Pract 2017;12:78-83.

9. Tinkle S, McNeil SE, Mühlebach S, et al. Nanomedicines: addressing the scientific and regulatory gap. Ann New York Acad Sci 2014;1313:35-56.

10. Mohan SK, Veeraraghavan VP and Jainu M. Effect of pioglitazone, quercetin and hydroxy citric acid on extracellular matrix components in experimentally induced non-alcoholic steatohepatitis. Iranian J Basic Med Sci 2015;8(8):832-836.

11. Colombo M, Raposo G and Théry C. Biogenesis, secretion, and intercellular interactions of exosomes and other extracellular vesicles. Annu Rev Cell Dev Biol 2014;30:255-289.

12. Pucci $C$, Martinelli $C$ and Ciofani G. Innovative approaches for cancer treatment: current perspectives and new challenges. Ecancermedicalscience 2019;13:961.

13. Alasvand N, Urbanska AM, Rahmati M, et al. Therapeutic Nanoparticles for Targeted Delivery of Anticancer Drugs. Multifunctional Systems for Combined Delivery, Biosensing and Diagnostics 2017;12(2):23-31.

14. Menon A, Gayathri R. Preliminary Phytochemical Analysis And Cytotoxicity Potential Of Pineapple Extract On Oral Cancer Cell Lines. Asian J Pharm Clin Res 2016;6(4):67-72.

15. Rengasamy G, Jebaraj DM, Veeraraghavan VP, et al. Characterization, Partial Purification of Alkaline Protease from Intestinal Waste of Scomberomorus Guttatus and Production of Laundry Detergent with Alkaline Protease Additive. Indian J Pharma Edu Res 2016;50(2):S59-s67.

16. Wu F, Zhu J, Li G, et al. Biologically synthesized green gold nanoparticles from Siberian ginseng induce growth-inhibitory effect on melanoma cells (B16). Artif Cells Nanomed Biotech 2019; 47(1):3297-3305.

17. Ke Y, Al Aboody MS, Alturaiki W, et al. Photosynthesized gold nanoparticles from Catharanthus roseus induces caspase-mediated apoptosis in cervical cancer cells (HeLa). Artif Cells Nanomed Biotech 2019; 47(1).

18. Ma Y, Karunakaran T, Veeraraghavan VP, et al. Sesame Inhibits Cell Proliferation and Induces Apoptosis through Inhibition of STAT-3 Translocation in Thyroid Cancer Cell Lines (FTC-133). Biotech Bioproc Engi 2019;34:112-121.

19. Wang Y, Zhang Y, Guo Y, et al. Synthesis of Zinc oxide nanoparticles from Marsdenia tenacissima inhibits the cell proliferation and induces apoptosis in laryngeal cancer cells (Hep-2). J Photochem Photobiol B: Biology 2019; 201:111624.

20. Maeda $\mathrm{H}$ and Khatami M. Analyses of repeated failures in cancer therapy for solid tumors: poor tumor-selective drug delivery, low therapeutic efficacy and unsustainable costs. Clin Transl Med 2018; 7(1):11..

21. Krzyszczyk P, Acevedo A, Davidoff EJ, et al. The growing role of precision and personalized medicine for cancer treatment. Sci Tech 2018;6(3-4):79-100.

22. Bilkey GA, Burns BL, Coles EP, et al. Optimizing Precision Medicine for Public Health. Front Public Health 2019; 7: 42.

23. Re A, Nardella C, Quattrone A, et al. Editorial: Precision Medicine in Oncology. Front Oncol 2018;8:479.

24. Marson FAL. Personalized or Precision Medicine? The Example of Cystic Fibrosis. Front Pharmacol 2017;8:390.

25. Iriart JAB. Precision medicine/personalized medicine: a critical analysis of movements inthe transformation of biomedicine in the early 21 st century. Cadernos de Saude Publica 2019;35(3):e00153118.

26. Verma M. Personalized Medicine and Cancer. J Personal Med 2012; 2(1): 1-14.

27. Rosen ST. Why Precision Medicine Continues to Be the Future of Health Care. Oncology Times 2017;32)4):34-42.

28. Bettaieb A, Paul C, Plenchette S, et al. Precision medicine in breast cancer: reality or utopia? J Transl Med 2017;15(1):139.

29. Jeibouei S, Akbari ME, Kalbasi A, et al. Personalized medicine in breast cancer: pharmacogenomics approaches. Pharmacogen Personal Med 2019;12: 59-73.

30. Yau TO. Precision treatment in colorectal cancer: Now and the future. JGH Open 2019; 3(5):361-369.

31. Brown NA, Aisner DL and Oxnard GR. Precision Medicine in Non-Small Cell Lung Cancer: Current Standards in Pathology and Biomarker Interpretation. Am Soc Clin Oncol Ed Book 2018.

32. Li Z, Veeraraghavan VP, Mohan SK, et al. Apoptotic induction and anti-metastatic activity of eugenol encapsulated chitosan nanopolymer on rat glioma C6 cells via alleviating the MMP signaling pathway. J Photochem and Photobiol B: Biol 2020;203:111773

33. Ponnulakshmi R, Shyamaladevi B, Vijayalakshmi P, et al. In silico and in vivo analysis to identify the antidiabetic activity of beta sitosterol in adipose tissue of high fat diet and sucrose induced type-2 diabetic experimental rats. Toxicol Mechan Meth 2019;29(4):276-290. 